Check for updates

1 London

2 The BMJ

Cite this as: BMJ 2021;375:n2938 http://dx.doi.org/10.1136/bmj.n2938 Published: 26 November 2021

\section{Tom Nolan’s research reviews-26 November 2021}

\author{
Tom Nolan GPclinical editor, 2
}

\section{Thiazides and $\beta$ blockers feel the pressure}

“5 mm Hg, 11\%." I'm trying to commit these numbers to memory for consultations with patients at high risk of developing diabetes: lowering your systolic blood pressure by five points reduces the chances of getting diabetes by about a tenth. This was one of the conclusions of a meta-analysis of individual participant data from 22 studies conducted between 1973 and 2008.

The study also found that ACE inhibitors and angiotensin II receptor blockers reduce the risk of developing diabetes (relative risk of 0.84 for both drug classes), whereas thiazide diuretics and $\beta$ blockers increase the risk (relative risks of 1.2 and 1.48 respectively). Calcium channel blockers, which have always seemed such stoical drugs, sit in the middle refusing to get involved (relative risk 1.02). So we now have another factor to throw into the pot when discussing the choice of antihypertensive with patients. Although age and ethnicity are central to NICE recommendations on choice of antihypertensive, these weren't discussed in this paper.

\section{Glargine battles on as insulin turns 100}

The Lancet is celebrating the 100 year anniversary of the isolation and purification of insulin in style, by treating us to a head-to-head battle between insulin glargine and one of the new kids on the block: tirzepatide. And while there was no knockout blow from tirzepatide, it definitely won on points.

This was an open label study across 14 countries, of 2002 people with type 2 diabetes and inadequate glycaemic control who were already taking metformin, sulfonylureas, and/or a sodium-glucose cotransporter-2 inhibitor and were at high cardiovascular risk (such as already having had a cardiovascular event). ${ }^{2}$ They were randomly assigned to tirzepatide (a dual GIP and GLP-1 receptor agonist) or insulin glargine. After a year, average $\mathrm{HbA1c}$ improvements with tirzepatide were $10.9 \mathrm{mmol} / \mathrm{mol}$ (0.99\% in old money) greater than those seen with glargine. The HbA1c changes were $-24.5 \mathrm{mmol} / \mathrm{mol}$ $(-2.24 \%)$ with $5 \mathrm{mg}$ tirzepatide versus $-15.7 \mathrm{mmol} / \mathrm{mol}$ $(-1.44 \%)$ with insulin glargine. Upping the tirzepatide dose seemed to give only marginal extra benefit: -28.2 $\mathrm{mmol} / \mathrm{mol}(-2.58 \%)$ with $15 \mathrm{mg}$. There was no difference in cardiovascular events or deaths between the two drugs. Those taking tirzepatide lost an average of $7.1 \mathrm{~kg}$, but gastrointestinal side effects were common: nausea and diarrhoea each occurred in about a fifth of participants.

\section{Plasma's going home}

Convalescent plasma from blood donors who have recovered from covid-19 was an early hope for an effective treatment. But, when given to high risk patients attending emergency departments within seven days of symptom onset, it doesn't seem to prevent symptom progression, according to a single blinded, randomised placebo-controlled trial in the US. ${ }^{3}$ The study was halted early because the "stopping threshold for futility had been reached." Sports fans will know this as the moment when your team is losing so badly you turn to your neighbour and say, "This is pointless, I'm going home." But it might not be game over for convalescent plasma. The study's authors suggest further research may find it can have a role if administered before the development of native antibodies, for preventing symptomatic covid-19 after exposure, or even as an early therapeutic agent during the early phases of another pandemic.

\section{Sacubitril-valsartan swings again}

Sacubitril-valsartan is back in another major journal after last week's disappointing findings in patients with acute myocardial infarction complicated by heart failure. In the spotlight this week are patients with heart failure and a left ventricular ejection fraction $>40 \%$, elevated $\mathrm{N}$-terminal pro-brain natriuretic peptide (NT-proBNP) levels, structural heart disease, and reduced quality of life. The randomised controlled trial found no benefits in quality of life, mobility (six minute walk distance), or New York Heart Association classification after 24 weeks compared with controls (enalapril, valsartan, or placebo, depending on prior use of a renin angiotensin system inhibitor). ${ }^{4}$ Those in the sacubitril-valsartan arm did have lower NT-proBNP levels at 12 weeks, but it remains to be seen if this translates to improvements in symptoms or mortality over the long term.

\section{The sound of silence}

A research letter in JAMA Internal Medicine about inaudible heart sounds sent me down a Google rabbit hole that ended at chapter 42 of Common Clinical Presentations in Dogs and Cats (definitely one for the Christmas list). ${ }^{5}$ Apparently, quiet heart sounds in dogs are often due to neoplastic or idiopathic disease, whereas in cats congestive heart failure secondary to cardiomyopathy is more common. Returning to humans, the research letter presents an analysis of heart sound recordings from 200 hospitalised patients who were undergoing echocardiography. They found that "heart sounds were clinically undetectable at the aortic location in $30(15 \%)$ patients and at the mitral location in 130 (65\%) patients.” Of the 66 patients with mitral regurgitation, $62 \%$ had undetectable heart sounds. The numbers seem high, and there are various methodological limitations, but it's good to read some research that seeks to question and advance the clinical value of examination findings. 


\section{OPINION}

Competing interests: none declared

Provenance and peer review: commissioned, not peer reviewed

1 Nazarzadeh M, Bidel Z, Canoy D, etalBlood Pressure Lowering Treatment Trialists' Collaboration Blood pressure lowering and risk of new-onset type 2 diabetes: an individual participant data meta-analysis. Lancet2021;398:1803-10. doi: 10.1016/S0140-6736(21)01920-6. pmid: 34774144

2 Del Prato S, Kahn SE, Pavo I, etalSURPASS-4 Investigators. Tirzepatide versus insulin glargine in type 2 diabetes and increased cardiovascular risk (SURPASS-4): a randomised, open-label, parallel-group, multicentre, phase 3 trial. Lancet 2021;398:1811-24.

doi: 10.1016/S0140-6736(21)02188-7. pmid: 34672967

3 Korley FK, Durkalski-Mauldin V, Yeatts SD, etalSIREN-C3PO Investigators. Early Convalescent Plasma for High-Risk Outpatients with Covid-19. N Engl J Med 2021;385:1951-60. doi: 10.1056/NEJMoa2103784. pmid: 34407339

4 Pieske B, Wachter R, Shah SJ, etalPARALLAX Investigators and Committee members. Effect of Sacubitril/Valsartan vs Standard Medical Therapies on Plasma NT-proBNP Concentration and Submaximal Exercise Capacity in Patients With Heart Failure and Preserved Ejection Fraction: The PARALLAX Randomized Clinical Trial. JAMA 2021;326:1919-29. doi: 10.1001/jama.2021.18463. pmid: 34783839

5 Jariwala N, Czako S, Brenton L, etal. Clinically Undetectable Heart Sounds in Hospitalized Patients Undergoing Echocardiography. JAMA Intern Med

2021;e216594doi: 10.1001/jamainternmed.2021.6594. pmid: 34779823 